\title{
ASCL2 expression contributes to gastric tumor migration and invasion by downregulating miR223 and inducing EMT
}

\author{
QINGSONG ZUO*, JIE WANG ${ }^{*}$, CHAO CHEN, YONG ZHANG, \\ DIAN-XU FENG, RONGHUA ZHAO and TENG CHEN \\ Department of General Surgery, Putuo Hospital, Shanghai University of \\ Traditional Chinese Medicine, Shanghai 200062, P.R. China
}

Received May 15, 2017; Accepted November 23, 2017

DOI: $10.3892 / \mathrm{mmr} .2018 .9363$

\begin{abstract}
Achaete-scute homolog 2 (ASCL2), a basic helix-loop-helix transcription factor, serves an essential role in the maintenance of adult intestinal stem cells and the growth of gastric cancer (GC). However, the function of ASCL2 in the metastasis of GC is poorly understood. The present study aimed to evaluate the effect of ASCL2 expression on gastric tumor metastasis. ASCL2 protein expression was detected in 32 cases of gastric metastasis and its relevant primary tumors using western blotting and immunohistochemistry. The data suggested that the expression of ASCL2 was highest in metastatic tumors, among adjacent normal tissues, primary gastric tumors and gastric metastatic tumors. Furthermore, ASCL2-overexpressing GC cell lines MKN1-ASCL2 and SNU16-ASCL2 were established. An in vitro assay suggested that microRNA 223 (miR223) expression was downregulated following ASCL2 overexpression, and that the expression of the epithelium-associated protein E-cadherin was significantly decreased, while a series of mesenchyme-associated proteins, including zinc finger E-box-binding homeobox 1 (Zeb-1), twist-related protein 1, integrin, vimentin, $72 \mathrm{kDa}$ type IV collagenase and matrix metalloproteinase-9 were upregulated in ASCL2-overexpressing cells. Overexpression of miR223 attenuated the epithelial-mesenchymal transition (EMT)-promoting effect induced by ASCL2 expression. In addition, the results of the chromatin immunoprecipitation and luciferase reporter gene assays indicated that ASCL2 was able to interact with the promoter of pre-miR223, and to inhibit the maturation of miR223, which may interact with the 3' untranslated region of Zeb-1 and inhibit EMT in tumor cells. The
\end{abstract}

Correspondence to: Dr Teng Chen, Department of General Surgery, Putuo Hospital, Shanghai University of Traditional Chinese Medicine, 164 Lanxi Road, Shanghai 200062, P.R. China

E-mail: chenteng2017@163.com

${ }^{*}$ Contributed equally

Key words: achaete-scute homolog 2, microRNA epithelial-mesenchymal transition, gastric cancer, metastasis results of the present study demonstrated that ASCL2 was able to downregulate the expression level of miR223, contribute to EMT and promote gastric tumor metastasis, which indicated that ASCL2 may serve as a therapeutic target in the treatment of GC.

\section{Introduction}

Gastric cancer (GC) is the second most common cause of cancer-associated mortality in the world, and the incidence GC is highest in East Asia, Eastern Europe and parts of Latin America $(1,2)$. However, the precise mechanisms underlying gastric carcinogenesis are not yet completely understood (3). Therefore, it is important to identify the regulatory mechanisms and signaling pathways involved in GC.

The achaete-scute homolog 2 (ASCL2) gene is a member of the basic helix-loop-helix family of transcription factors, which are downstream target of Wnt signaling and initiate transcription by binding to the E-box (4). ASCL2 is involved in the determination of neuronal precursors in the peripheral and central nervous systems (5), and it is also a cancer stem cell marker (6); certain reports have revealed that ASCL2 promotes cell growth and migration in colon cancer $(7,8)$. However, the function of ASCL2 in the metastasis of GC is poorly understood.

MicroRNAs (miRNAs/miRs) are a class of endogenous small non-coding RNAs with maximum length of $25 \mathrm{bp}(8,9)$. At present, $>1,000$ types of miRNA have been identified in the human body (10). miRNAs are involved in a number of physiological and pathological processes, including cell proliferation, differentiation, apoptosis, migration, invasion, organ development and tumor development $(11,12)$. miRNAs primarily affect the post-transcriptional level of gene coding, and may inhibit or promote the expression of target genes. In tumorigenesis, miRNAs may act as tumor suppressor genes or carcinogenic genes (13). miR223 has been demonstrated to reverse epithelial-mesenchymal transition (EMT), and to inhibit the migration and invasion of melanoma cells by inducing the expression of E-cadherin protein (14). EMT refers to the process of transition between epithelial cells and mesenchymal cells under certain physiological or pathological conditions, which contributes to the occurrence and development of tumors, particularly invasion and metastasis (8). EMT 
has been demonstrated to serve a critical role in gastric tumor migration (15); therefore, it is important to reveal the molecular mechanism underlying EMT, and its effect on the invasion and metastasis of gastric tumors.

In the present clinical study, it was observed that ASCL2 was upregulated in metastatic tumors compared with primary tumors of patients. The present study investigated the effect of ASCL2 on the invasion and migration of GC cells, and the underlying mechanism.

\section{Materials and methods}

Sample collection. A total of 32 cases of gastric tumor and their relevant metastases were obtained from patients, following the provision of written informed consent. The patients were 41-91 years old and the male/female radio was 1.47 . The samples were collected between March 2009 and June 2014. The procedure was approved by the ethical committee of Putuo Hospital Affiliated to Shanghai University of Traditional Chinese Medicine (Shanghai, China).

Cell culture. Human gastric carcinoma cell lines MKN-1 and SNU16 were obtained from the American Type Culture Collection (Manassas, VA, USA) and were maintained in RPMI-1640 medium (Sigma-Aldrich; Merck KGaA, Darmstadt, Germany) containing $10 \%$ fetal bovine serum (FBS; HyClone; GE Healthcare Life Sciences, Logan, UT, USA) at $37^{\circ} \mathrm{C}$ and $5 \% \mathrm{CO}_{2}$, with the medium changed every two days. Cells were passaged at $80 \%$ confluence and seeded at $30 \%$ confluence for maintenance of optimal proliferating conditions.

Immunohistochemistry. Immunohistochemical analysis was performed to evaluate the ASCL2 expression in the adjacent tissues (3 $\mathrm{cm}$ next to the tumor), primary gastric tumor or metastases. Following deparaffinization and rehydration with $\mathrm{ddH}_{2} \mathrm{O}$ at room temperature, the $10 \%$ formalin-fixed paraffin-embedded tumor tissue sections $(4 \mu \mathrm{m})$ were incubated with $3 \%$ hydrogen peroxide in methanol to quench endogenous peroxidase activity. The sections were blocked for $30 \mathrm{~min}$ with $1 \%$ bovine serum albumin (Sangon Biotech Co., Ltd., Shanghai, China) and incubated with the primary antibodies ASCL2 (ab116699; 1:100; Abcam, Cambridge, UK) at $4^{\circ} \mathrm{C}$ overnight. The sections were washed with PBS and incubated with horseradish peroxidase (HRP)-conjugated secondary antibody (ab205718; 1:100; Abcam) at room temperature for $1 \mathrm{~h}$. The products were visualized using a diaminobenzidine staining kit (Tiangen Biotech Co., Ltd., Beijing, China) at room temperature for $2 \mathrm{~min}$ and counterstained with hematoxylin at room temperature for $5 \mathrm{~min}$. The images were obtained by microscope (IX71; Olympus Corporation, Tokyo, Japan).

ASCL2 overexpression assay. Lentivirus particles expressing ASCL2 were produced by Shanghai GeneChem Co., Ltd (Shanghai, China). MKN-1 and SNU16 cells (5x10 4 each) were inoculated in 12-well plates and incubated in complete medium with $1 \times 10^{8} \mathrm{TU} / \mathrm{ml}$ of lentivirus. At $24 \mathrm{~h}$ following seeding, the medium was aspirated and replaced with $1 \mathrm{ml}$ fresh medium overnight. In order to obtain a stable transfected cell line, the lentivirus-infected cells were screened using culture medium containing $2 \mu \mathrm{g} / \mathrm{ml}$ puromycin, and the culture medium was changed every three days.

Cell transfection. Hsa-miR-223 mimic (UGUCAGUUU GUCAAAUACCCC) and negative control mimic (UCA CAACCUCCUAGAAAGAGUAGA) were purchased from Guangzhou RiboBio Co., Ltd. (Guangzhou, China). The cells at $60 \%$ confluence (determined by blood counting chamber) were seeded in 6-well plates and were transfected with hsa-miR-223 or negative control mimic at a final concentration of $100 \mathrm{nM}$ using Lipofectamine ${ }^{\circledR} 2000$ (Invitrogen; Thermo Fisher Scientific, Inc., Waltham, MA, USA), according to the manufacturer's protocol. Cells were collected $48 \mathrm{~h}$ post-transfection for reverse transcription-quantitative polymerase chain reaction (RT-qPCR) analysis, western blotting or Transwell assays.

Western blot analysis. Cell lysates or the homogenized tissues from tumor xenografts were dissolved in RIPA lysis buffer (Beyotime Institute of Biotechnology, Haimen, China), protein concentration was determined by BCA method, and lysates were separated by $10 \%$ SDS-PAGE and transferred to a nitrocellulose membrane, and GAPDH was used as a control. Following blocking with 5\% dried skimmed milk for $2 \mathrm{~h}$ in room temperature, the membrane was probed with specific primary antibodies ASCL2 (ab116699), Zeb-1 (ab203829), E-cadherin (ab76055), Integrin (ab133557), Twist (ab50581), Vimentin (ab8978), MMP-2 (ab92536), MMP-9 (ab73734) and GAPDH (ab8245; all from Abcam and diluted at $1: 1,000)$ overnight at $4^{\circ} \mathrm{C}$, followed by incubation with HRP-conjugated secondary IgG $(\mathrm{H}+\mathrm{L})$ antibody (ab205718; 1:1,000; Abcam) at room temperature for $1 \mathrm{~h}$. The blots were processed with Immobilon ${ }^{\mathrm{TM}}$ western chemiluminescent HRP substrate (EMD Millipore, Billerica, MA, USA) and analyzed with a gel imaging analysis system and Image Lab version 2.0.1 (Bio-Rad Laboratories, Inc., Hercules, CA, USA).

Migration assay. MKN-1 and SNU16 cells and their transfectants, at 90-100\% confluence in 6-well plates, were cultured overnight in serum-free medium. The medium was replaced with PBS, and the monolayers were wounded mechanically using a $10 \mu$ l Axygen pipette tip (Axygen Scientific, Inc., Union City, CA, USA). Following wounding, cells were rinsed twice with sterilized PBS and incubated in RPMI-1640 medium containing $10 \% \mathrm{FBS}$ at $37^{\circ} \mathrm{C}$, under $5 \% \mathrm{CO}_{2}$, and digital images of the healing conditions were obtained by microscope (IX71; Olympus Corporation, Tokyo, Japan) at 0 and $16 \mathrm{~h}$.

Transwell assay. The invasive capacity of the two types of GC cells overexpressing ASCL2 was evaluated by Transwell assay in a 24-well plate. Matrigel (BD Biosciences, Franklin Lakes, $\mathrm{NJ}$, USA) was diluted with $4^{\circ} \mathrm{C}$ pre-cooled serum-free medium to a final concentration of $1 \mathrm{mg} / \mathrm{ml}$. The upper chambers were coated with $100 \mu \mathrm{l}$ diluted Matrigel. For the stably-transfected cells, $5 \times 10^{3} / 200 \mu \mathrm{l}$ cells were seeded in the upper chambers and fed with serum-free medium, and $500 \mu 1$ medium with $20 \%$ FBS was added to the lower chambers. The cells were allowed to invade the Matrigel matrix for 8-24 h. The cells on the upper chamber were scraped off with a cotton swab and the transmigrated cells were fixed with $4 \%$ formaldehyde for 
$1 \mathrm{~h}$ at room temperature, washed with PBS 3 times and stained with $0.5 \%$ crystal violet for $15 \mathrm{~min}$, followed by washing with PBS for 3 times, and then counted in 5 randomly selected microscopic fields (magnification, x200). The cell numbers were counted by microscope, and all of the experiments were performed in triplicate.

RT-qPCR analysis. Total RNA was extracted using TRIzol reagent (Invitrogen; Thermo Fisher Scientific, Inc.), according to the manufacturer's protocol. First strand cDNA was synthesized using PrimeScript ${ }^{\mathrm{TM}}$ RT enzyme mix I, oligo dT primers and random hexamers (Takara Bio, Inc., Otsu, Japan). To determine the fold changes in each gene and miR, qPCR was performed using the first strand cDNA and the SYBR premix ExTaq $^{\mathrm{TM}}$ Green II (Takara Bio, Inc.). The primer sequences were as follows: ASCL2 forward, AACTTGAGCTGCTGG AGGGACA and reverse, TCTTGGCCAGCATGGAAAACT C; $\beta$-actin forward, CTGGAACGGTGAAGGTGACA, reverse, CGGCCACATTGTGAACTTTG; hsa-miR-223-3p mimic forward, UGUCAGUUUGUCAAAUACCCCA and reverse, UGGGGUAUUUGACAA ACUGACA; hsa-miR-141-3p, Forward, TAACACTGTCTGGTAAAGATGG, Reverse, CAG TGCGTGTCGTGGAGT; hsa-miR-137, Forward, TTATTG CTTAAGAATACGCGTAG, Reverse, CAGTGCGTGTCG TGGAGT; miRNA mimic negative control forward, UUU GUACUACACAAAAGUACUG and reverse, CAGUACUUU UGUGUAGUACAAA. The Primers of the other miRNAs were purchased from Guangzhou RiboBio Co., Ltd. Reaction and signal detection were measured using a qPCR system (Bio-Rad Laboratories, Inc.). The obtained cDNA was amplified using following procedure: $94^{\circ} \mathrm{C}(5 \mathrm{~min})$, then 30 cycles at $94^{\circ} \mathrm{C}(30 \mathrm{sec}), 59^{\circ} \mathrm{C}(30 \mathrm{sec})$ and $72^{\circ} \mathrm{C}(30 \mathrm{sec})$. Expression levels were calculated as the relative expression ratio using the $2^{-\Delta \Delta C q}$ method with $\beta$-actin as a reference. The qPCR was performed in triplicate independently.

Prediction and detection of EMT-associated protein binding to miR223. EMT-associated proteins binding to miR223 were predicted using TargetScan (www.targetscan.org). A total of 412 transcripts were predicted to be the targets of miR223; the cumulative weighted context ++ score of zinc finger E-box-binding homeobox 1 (Zeb-1) was 0.21 , total context ++ score was -0.21 , and the aggregate probability of conserved targeting was 0.47 . The present study examined all the potential targets which may be involved in EMT, migration and invasion by western blotting.

Chromatin immunoprecipitation (ChIP) assay. ChIP assays were performed using a ChIP assay kit (Upstate Biotechnology, Inc., Lake Placid, NY, USA), according to the manufacturer's protocol. Soluble chromatin was prepared from cells overexpressing ASCL-2 and control cells. Chromatin was immunoprecipitated with an antibody against ASCL2 (ab116699, Abcam, UK), which was diluted at $1: 100$ at $4^{\circ} \mathrm{C}$. The final DNA extracts were amplified by qPCR using primer pairs in the human pre-miR223 promoter according to the protocol described above. Pre-miR223 primer sequences: Forward primer: 5'-CCACGCTCCGTG TATTTGAC-3'. Reverse primer: 5'-CCGCACTTGGGGTAT TTGAC-3'.
Luciferase reporter assay. Fragments of the pre-miR223 promoter 5'-flanking sequence or the 3' untranslated region (UTR) sequences of Zeb-1 were amplified using PCR and cloned into the luciferase reporter vector pGL3-Basic (Promega Corporation, Madison, WI, USA). The day prior to transfection, the cells were plated in 24-well plates at a density of $5 \times 10^{4}$ cells/well. The pre-miR 223 promoter-luciferase constructs or the 3' UTR luciferase constructs of Zeb-1 were transfected into the cells using Lipofectamine ${ }^{\circledR} 2000$ (Invitrogen; Thermo Fisher Scientific, Inc.). To normalize for transfection efficiency, the cells were simultaneously co-transfected with a pRLTK vector expressing the Renilla luciferase enzyme (pRL; Promega Corporation). The cells were harvested following $24 \mathrm{~h}$, and the luciferase activity was measured using the Dual Luciferase Reporter Assay System (Promega Corporation) with a single sample luminometer. In a similar way, the cells were transfected with the pRL-TK vector. Pre-miR223 activity is presented as the percentage of pGL3-control activity.

Statistical analysis. Data are expressed as the mean \pm standard deviation of three different experiments. The Student's t-test was used to analyze the comparisons between two groups, and analysis of variance was used to analyze the comparisons between multiple groups and followed by Newman-Keuls post hoc comparison test with differences. The statistical significance of the results was evaluated using SPSS version 17.0 (SPSS, Inc., Chicago, IL, USA). $\mathrm{P}<0.05$ was considered to indicate a statistically significant difference.

\section{Results}

Expression of ASCL2 is highest in metastases, among adjacent normal tissues, primary gastric tumors and gastric metastases. In order to study the expression level of ASCL2 in different parts of GC, protein and RNA were extracted from 32 cases of GC adjacent normal tissues, the primary gastric tumor and metastatic cancer tissue, and the expression of ASCL2 was analyzed using western blotting, qPCR and immunohistochemistry (Fig. 1). The expression of ASCL2 protein in metastatic tissues was highest, and was at its lowest in normal tissues (Fig. 1A and C), and the mRNA level of ASCL2 in metastatic tissues of GC was significantly higher compared with that in normal tissues (Fig. 1B), which was consistent with the results of the western blotting and immunohistochemistry. It was suggested that the high expression of ASCL2 may be associated with the metastasis of GC cells in metastatic GC tissues.

ASCL2 expression contributes to cell migration and invasion in MKN-1 and SNU16 cells. In order to further study the role of ASCL2 in the metastasis of GC, the ASCL2 plasmid was transfected into MKN-1 and SNU16 cells to construct ASCL2-overexpressing stably-transfected cell lines (Fig. 2A). Transwell experiments and wound healing assays were performed to study the effect of ASCL2 on GC metastasis. From the wound healing assay, the scratch width in ASCL2 overexpression and NC group cells was detected at $16 \mathrm{~h}$. The scratches in the NC group were significantly 
A

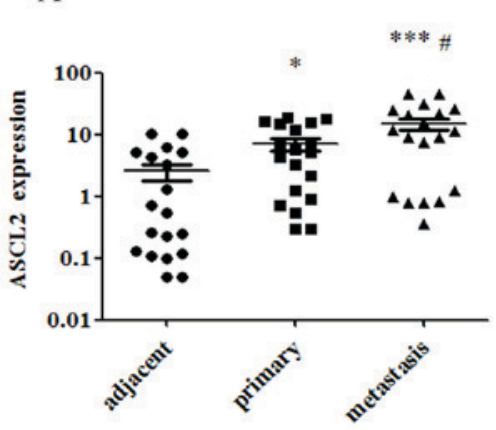

B

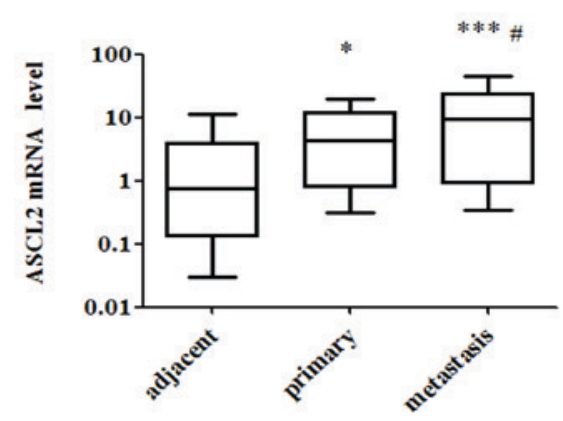

C

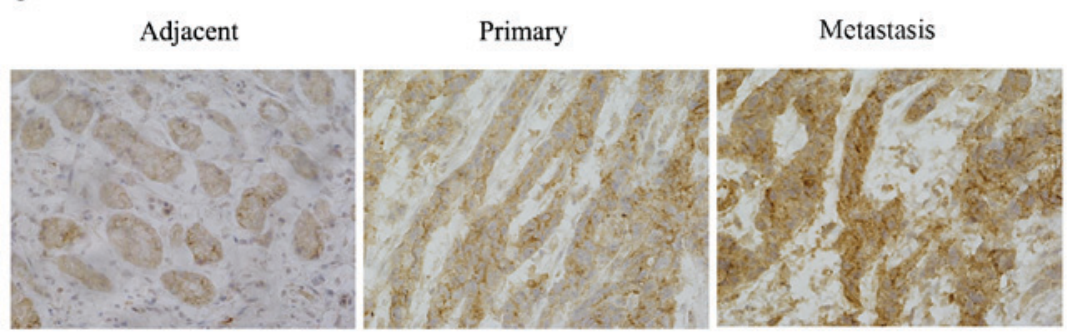

Figure 1. Expression of ASCL2 is highest in metastases, among adjacent normal tissues, primary gastric tumors and gastric metastases. (A) The expression of ASCL2 was detected in adjacent normal tissues, primary tumors and metastases by western blotting. (B) The mRNA expression level of ASCL2 was detected in adjacent normal tissues, primary tumors and metastases by quantitative polymerase chain reaction. (C) An immunohistochemistry assay evaluated the ASCL2 expression in the adjacent tissues, primary gastric tumors and metastases (magnification, $\mathrm{x} 400$ ). ${ }^{*} \mathrm{P}<0.05,{ }^{* * * *} \mathrm{P}<0.001$ vs. adjacent group; ${ }^{*} \mathrm{P}<0.05$ vs. primary group. ASCL2, achaete-scute homolog 2 .

wider compared with the ASCL2 overexpression group (Fig. 2B), which indicated that overexpression of ASCL2 was able to increase cell migration and invasion. In addition, a Transwell experiment was performed, the results of which demonstrated that the number of MKN-1 and SNU16 cells in the ASCL2 overexpression group was significantly increased compared with the NC group (Fig. 2C), indicating that the overexpression of ASCL-2 significantly increased the invasive ability of MKN-1 and SNU16 cells. From the above results, it was demonstrated that ASCL2 had a role in promoting the migration and invasion of GC cells, although the mechanism regulating the metastasis of GC via ASCL-2 remained unclear.

ASCL2 promotes the expression of EMT-inducing proteins. Since ASCL2 was able to promote the metastasis of GC cells, the migration of cells was associated with the dynamic alterations in adhesion between cytoskeletal proteins and the cell matrix. In this process, EMT-inducing proteins served an important role; therefore, it was hypothesized that ASCL2 may regulate EMT-associated proteins during cell migration. To this end, proteins were extracted from cells and western blotting was performed. From the experimental results, it was observed that the expression of E-cadherin in ASCL2-overexpressing cell lines decreased, while Zeb-1, twist-related protein 1 (Twist), Integrin, Vimentin, $72 \mathrm{kDa}$ type IV collagenase (MMP-2) and matrix metalloproteinase 9 (MMP-9) were upregulated in ASCL2-overexpressing cell lines (Fig. 3), which indicated that ASCL2 upregulated EMT-inducing protein expression and promoted cell migration. However, the mechanism underlying the regulatory effect of ASCL2 on EMT-associated proteins remained to be elucidated.
EMT-associated miRNAs alter following ASCL2 overexpression in MKN-1 and SNU16 cells. The present study demonstrated that miRNAs are an important switch in regulating gene expression and regulating various life phenomena, including tumor cell proliferation, differentiation, apoptosis and motility. It was hypothesized that EMT-associated proteins may be mediated by EMT-associated miRNAs. In order to study this hypothesis, RT-qPCR analysis was performed on the ASCL2 overexpressing cell lines. The results illustrated a significant reduction in miR223 out of a series of EMT-associated miRNAs (Fig. 4A), which indicated that ASCL2 regulated the expression of metastasis-associated proteins by downregulating miR223. Furthermore, the expression level of miRNA223 in the samples from patients was assessed by RT-qPCR analysis and the data suggested that the level of miR223 was at its lowest in metastatic tissues and highest in normal tissues (Fig. 4B).

Overexpressing miR223 attenuates the EMT-promoting effect induced by ASCL2 expression. In order to study the role of miR223 in the migration of GC cells, miR223 mimics and control mimics were overexpressed in MNK1 and SNU16 cells (Fig. 5A), and a Transwell assay was performed in which miR223 mimic was added to the ASCL2 overexpression group cells and the NC group cells, respectively (Fig. 5B). The miR223 mimic was able to reduce the number of penetrating cells in the ASCL2 overexpression and the NC groups (Fig. 5B), which indicated that ASCL2 may reduce the decreased cell migration induced by miR223. EMT-associated protein binding to miR223 was predicted using TargetScan (Fig. 5C). The results of the luciferase reporter assay suggested that miR223 was able to bind to the 3'UTR of Zeb-1 (Fig. 5D). 
A B

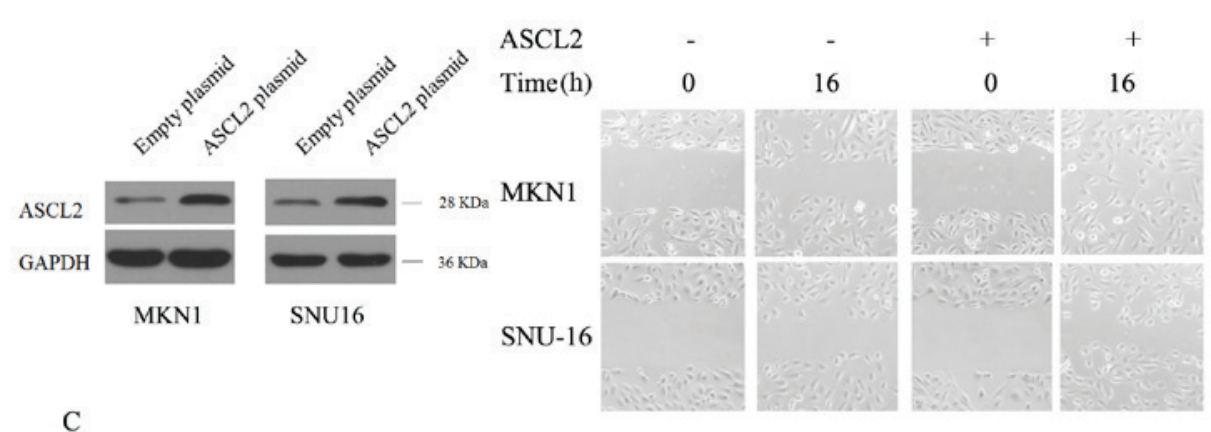

$\mathrm{C}$
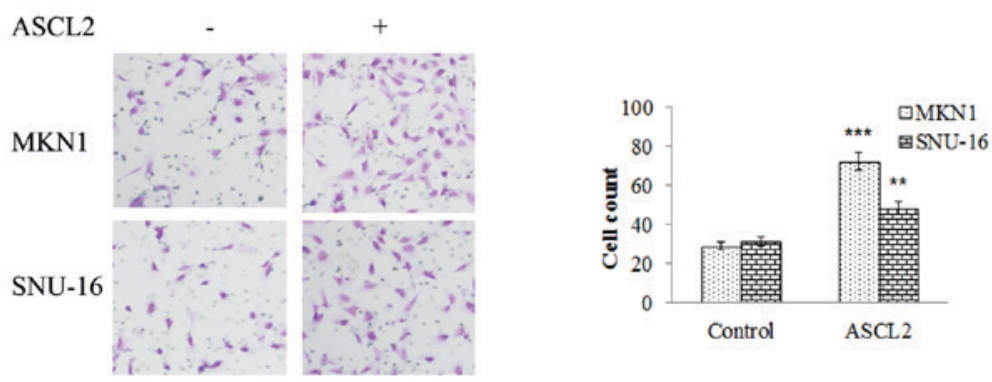

Figure 2. ASCL2 expression contributes to cell migration and invasion in MKN-1 and SNU16 cells. (A) The expression of ASCL2 was detected in control and ASCL2 overexpression cells. (B) The effect of ASCL2 expression on the migration of MKN-1 and SNU16 cells was accessed by wound healing assay (magnification, x200). (C) The effect of ASCL2 expression on the invasion of MKN-1 and SNU16 cells was evaluated by Transwell assay (magnification, $\mathrm{x} 200) .{ }^{* *} \mathrm{P}<0.01,{ }^{* * *} \mathrm{P}<0.001$ vs. respective control. ASCL2, achaete-scute homolog 2 .

The effect of miR223 on EMT-associated proteins in the NC and ASCL2 overexpression groups was additionally detected. From the western blotting results, it was inferred that miR223 reduced EMT-associated protein expression in the two groups; however, in the ASCL2 overexpression group, the reduction in the expression of EMT-associated proteins (Zeb-1, Twist, Integrin, Vimentin) and migration related proteins (MMP-2 and MMP-9) were less marked compared with the NC group (Fig. 5E), indicating that ASCL2 may weaken the biological function of miR223.

ASCL2 may interact with the promoter of pre-miR223, and inhibit the maturation of miR223. In order to examine the role of ASCL2 in the biological function of miR223, the interaction between ASCL2 and miR223 was analyzed using ChIP and luciferase reporter assays. The results demonstrated that ASCL2 interacted with pre-miR223 (Fig. 6A and B) to inhibit the transformation of pre-miR223 to mature miR223 (Fig. 6C).

\section{Discussion}

$\mathrm{GC}$ is the most common malignant tumor of the digestive tract. Due to the incidence of GC, its invasive and metastatic characteristics, clear clinical symptoms and low cure rate, GC ranks 4 th among malignant tumors in the world $(16,17)$. At present, the pathogenesis of GC had not been completely elucidated. The occurrence of GC originates from gastric epithelial stem cells; normal gastric epithelial stem cells develop gene mutations and thus promote the occurrence and development of tumors. A small number of tumor stem cell-like cells have been identified in GC tissues, which have infinite self-renewal, multi-directional differentiation and strong proliferative potential, and also promote the infiltration and recurrence of
GC. The mechanisms involved in the invasion and metastasis of GC cells required further study. In the present study, the expression of the ASCL2 gene was significantly increased in GC metastatic tissues, as demonstrated by western blotting and qPCR analysis, which suggested that high expression of ASCL2 may be associated with the metastasis of GC cells.

ASCL2 is a basic/helical helix transcription factor whose expression is confined to the LGr5-positive colonic basal ganglion cells of the placenta, and small- and large-intestinal crypts and crypt base columnar cells $(1,7)$. ASCL2 may interact with colorectal epithelial cells via the homeobox protein CDX-2 (CDX2) proximal promoter to repress the expression of CDX2 (18). It has been reported that CDX2 gene overexpression may promote GC cellular apoptosis. ASCL2 may promote colon cancer cell growth and migration, and the upregulation of ASCL2 by promoter demethylation may promote the growth of GC cells; the results of the present study suggested that ASCL2 may be involved in the metastasis of GC cells. In order to test this hypothesis, an ASCL2 gene overexpression system was constructed in vitro, and the effect of ASCL2 on the metastasis of GC cells was studied using wound healing and Transwell assays. From the wound healing assay, the migration of the ASCL2 overexpression and NC groups of MKN-1 and SNU16 cells were observed at $16 \mathrm{~h}$. The scratches in the $\mathrm{NC}$ group were significantly wider compared with those of the ASCL2 overexpression group; the scratches were partially-healed, although the NC group exhibited clear scratches, which indicated that overexpression of ASCL2 may increase cell migration ability. The results of the Transwell assay demonstrated that the number of MKN-1 and SNU16 cells in the NC group was significantly decreased compared with the ASCL2 overexpression group, which indicated that the overexpression of ASCL2 significantly increased the invasive 


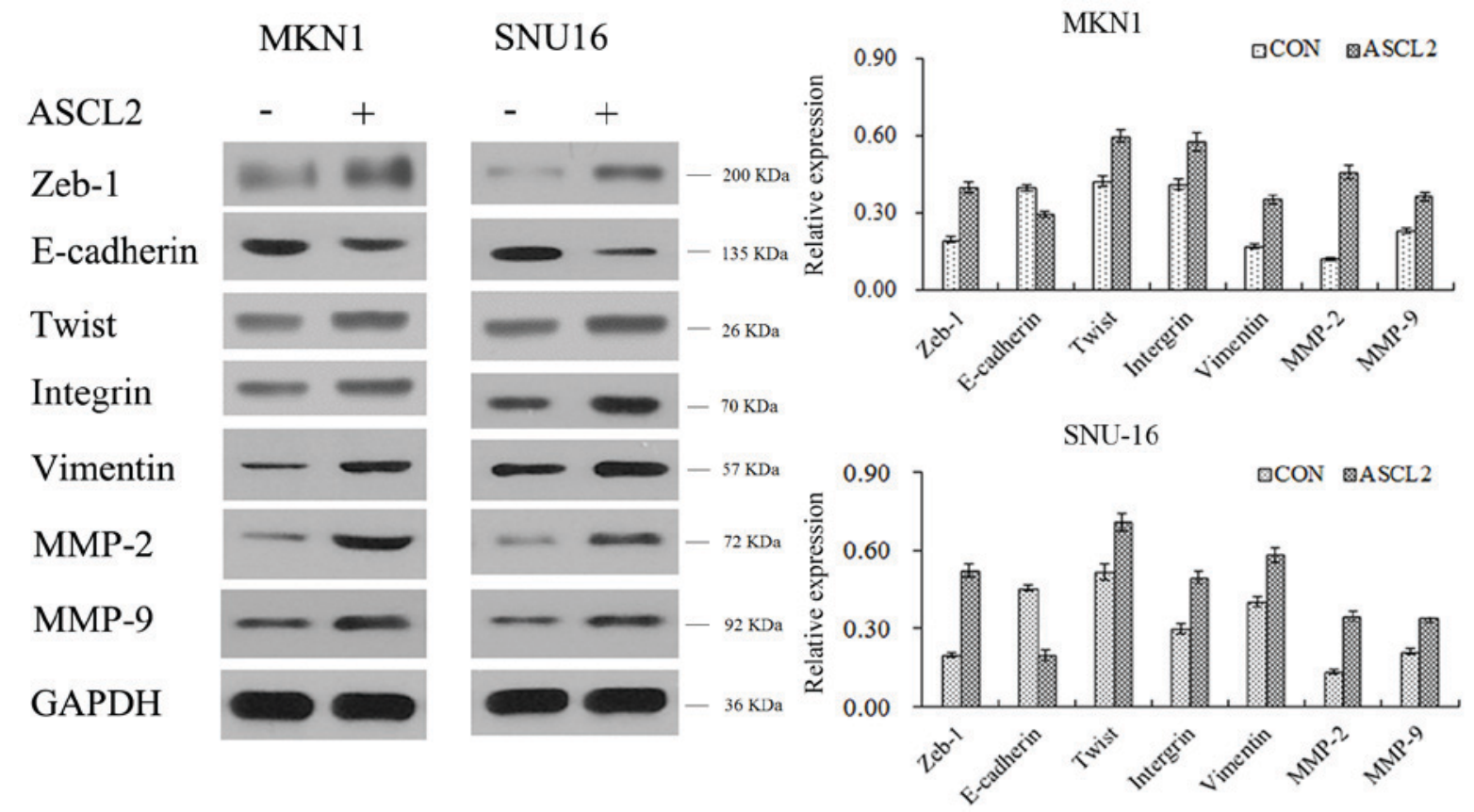

Figure 3. ASCL2 promotes the expression of epithelial-mesenchymal transition-inducing proteins. The expression of the epithelium-associated protein E-cadherin, and the mesenchyme-associated proteins Zeb-1, Twist, Integrin, Vimentin, MMP-2 and MMP-9 were detected by western blot analysis. ASCL2, achaete-scute homolog 2; Twist, twist-related protein 1; MMP-2, 72 kDa type IV collagenase; MMP-9, matrix metalloproteinase 9; Zeb-1, zinc E-box-binding homeobox 1; CON, control.

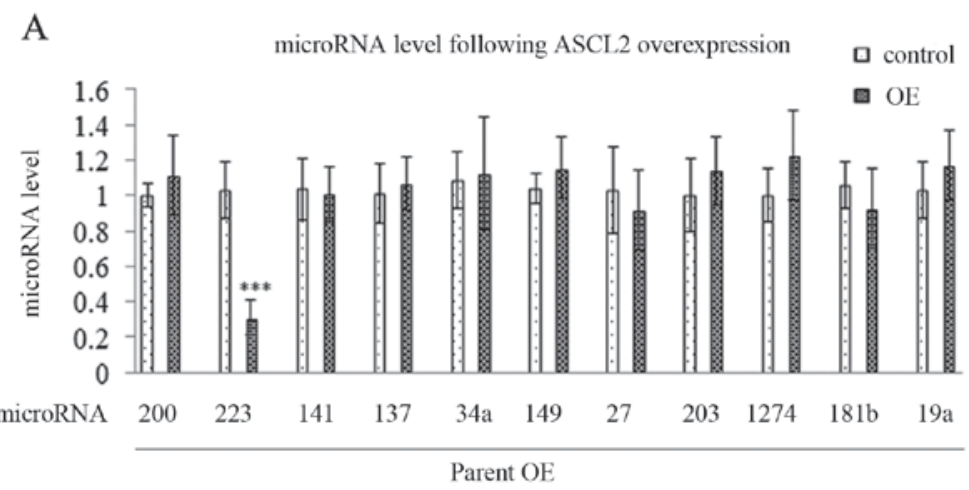

B

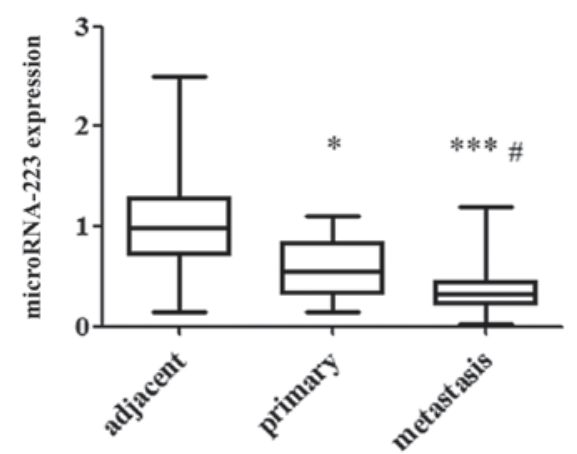

Figure 4. EMT-associated miRNAs alter following ASCL2 overexpression in MKN-1 and SNU-16 cells. (A) The expression levels of a series of miRNAs associated with EMT were detected by quantitative polymerase chain reaction. ${ }^{* * *} \mathrm{P}<0.001$ vs. control. (B) The levels of miRNA-223 were detected in the adjacent tissues, primary gastric tumors or metastases. ${ }^{*} \mathrm{P}<0.05,{ }^{* * * *} \mathrm{P}<0.001$ vs. adjacent group, ${ }^{*} \mathrm{P}<0.05$ vs. primary group. miRNA, microRNA; EMT, epithelial-mesenchymal transition; ASCL2, achaete-scute homolog 2. 
A

\section{B}
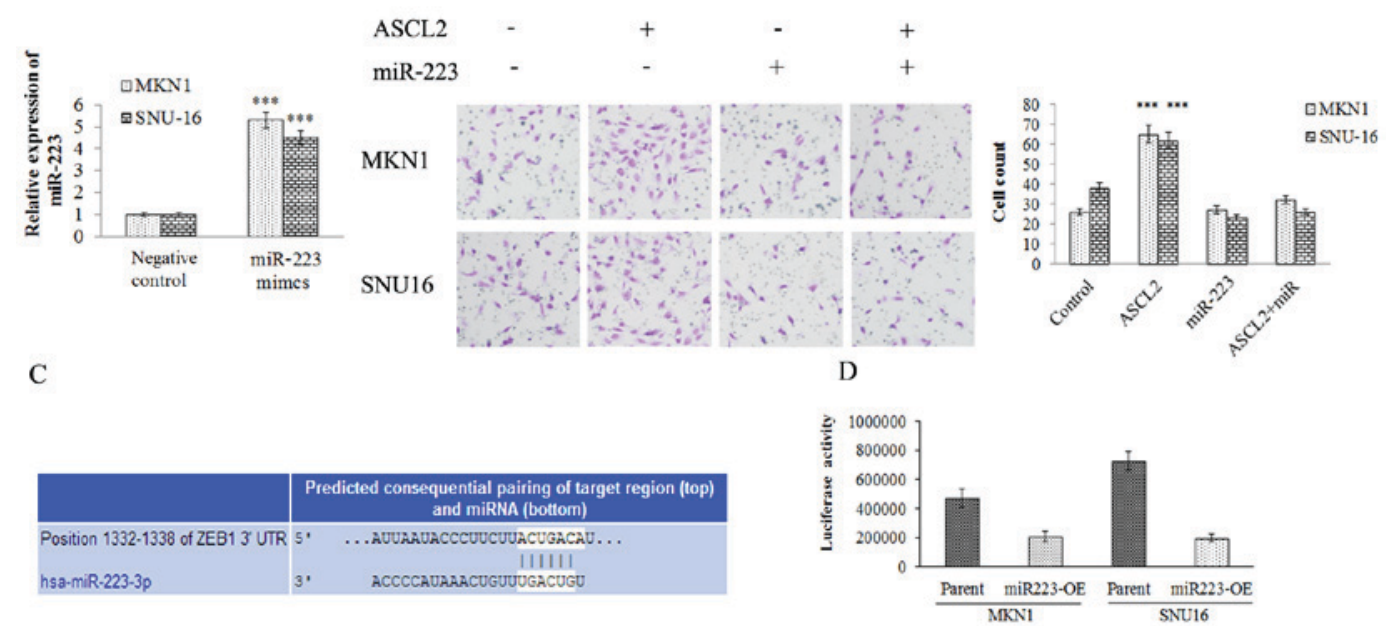

E
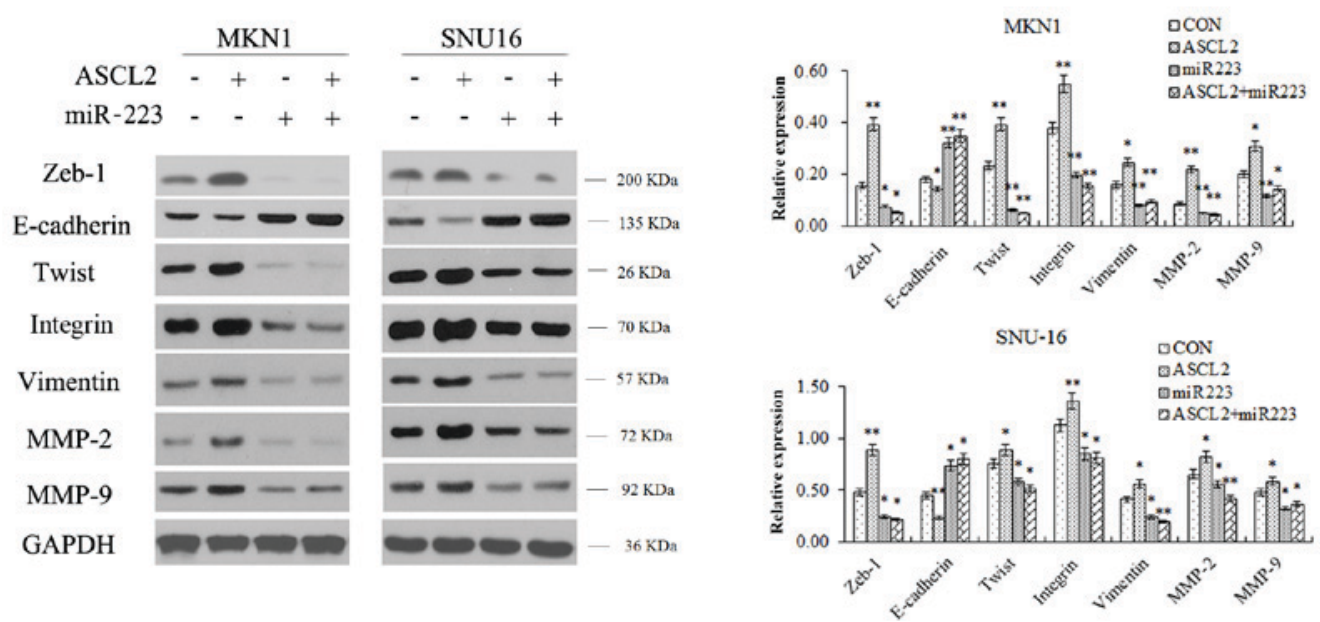

Figure 5. Overexpressing miR223 attenuates the EMT-promoting effect induced by ASCL2 expression. (A) The level of miR223 was detected by quantitative polymerase chain reaction. (B) The effect of miR223 on invasion in ASCL2-overexpressing MKN-1 and SNU16 cells was evaluated by Transwell assay (magnification, x200). (C) EMT-associated proteins binding to miR223 were predicted using TargetScan. (D) A luciferase reporter assay evaluated the binding of miR223 to the 3' UTR of Zeb-1. (E) The expression of the epithelium-associated protein E-cadherin, and the mesenchyme-associated proteins Zeb-1, Twist, Integrin, Vimentin, MMP-2 and MMP-9 was detected by western blot analysis. ${ }^{*} \mathrm{P}<0.05,{ }^{* * *} \mathrm{P}<0.01,{ }^{* * * *} \mathrm{P}<0.001$ vs. respective control. miR, microRNA; ASCL2, achaete-scute homolog 2; EMT, epithelial-mesenchymal transition; UTR, untranslated region; OE, overexpression; Twist, twist-related protein 1; MMP-2, 72 kDa type IV collagenase; MMP-9, matrix metalloproteinase 9; Zeb-1, zinc E-box-binding homeobox 1; CON, control.

ability of MKN-1 and SNU16 cells. From the above results, it was inferred that ASCL2 had promoted the migration and invasion of GC cells, although the mechanism regulating the metastasis of GC via ASCL2 remained unclear.

EMT is an important malignant biological behavior of tumors. The invasion and migration of tumor cells may be enhanced through EMT (19), and EMT serves an important role in secondary metastasis and promotes primary metastasis of gastric carcinoma, colon cancer, liver cancer, breast cancer, ovarian cancer and lung cancer (20). The role of EMT in tumor invasion and metastasis has become a research focus, and it was considered to be the initial step in tumor invasion and metastasis (21). During EMT, epithelial cells lose their epithelial characteristics, illustrated by the downregulation of E-cadherin, while acquiring a mesenchymal phenotype, characterized by the upregulation of mesenchymal proteins, including vimentin and N-cadherin. In GC, the upregulation of ASCL2 may promote GC metastasis. EMT is an important promoter of tumor metastasis; it was therefore hypothesized that ASCL2 may affect GC cell metastasis by affecting the EMT process. In order to study the association between ASCL2 and EMT, the alteration in EMT-associated protein expression following overexpression of ASCL2 was examined by western blotting. The results demonstrated that the expression of E-cadherin in ASCL2-overexpressing cell lines decreased, while Zeb-1, Twist, Integrin, Vimentin, MMP-2 and MMP-9 were upregulated in ASCL2-overexpressing cell lines, indicating that ASCL2 upregulated EMT-inducing proteins and promoted cell migration. However, the mechanism underlying the effect of ASCL2 on EMT-associated proteins has seldom been studied.

A previous study confirmed that miRNAs are associated with the occurrence, development, invasion and metastasis of GC, and they have become some of the specific molecular markers of GC (8). In tumorigenesis, miRNAs act as tumor suppressor genes or carcinogenic genes, and miR223 was able to reverse EMT, and inhibit the migration and invasion of melanoma cells induced the expression of E-cadherin protein. 
A

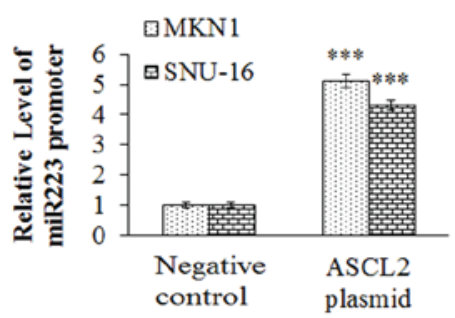

$\mathrm{C}$

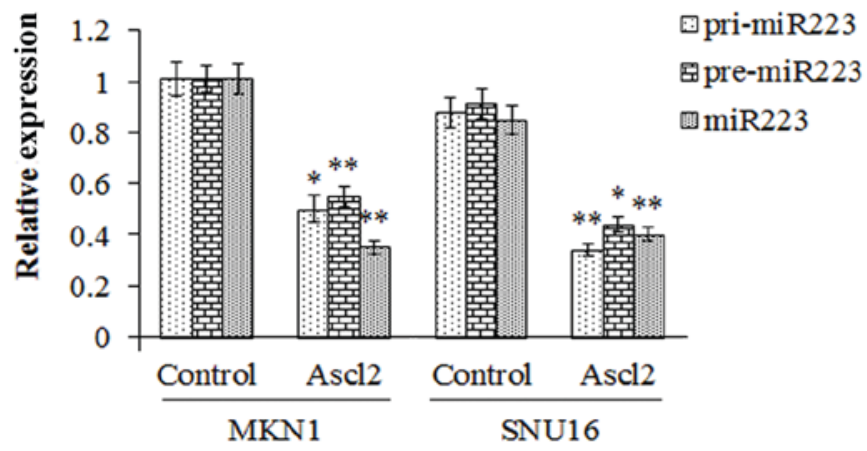

B

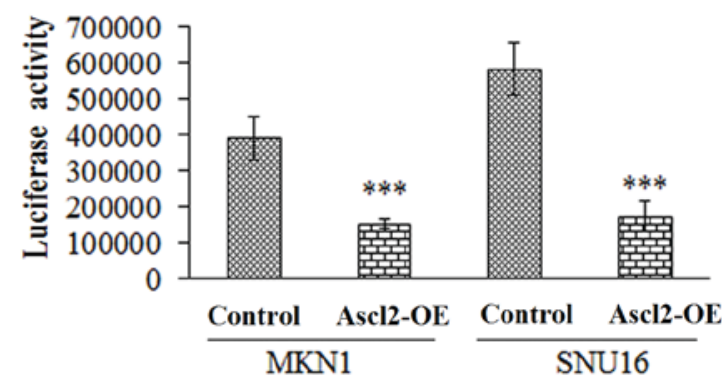

Figure 6. ASCL2 may interact with the promoter of pre-miR223 and inhibit the maturation of miR223. (A) The interaction between the promoter of pre-miR223 and ASCL2 was detected by chromatin immunoprecipitation assay. (B) The effect of ASCL2 on the activity of the promoter of pre-miR223 was detected by luciferase reporter assay. (C) The expression levels of pre-miR223, pri-miR223 and miR 223 were analyzed by quantitative polymerase chain reaction. ${ }^{*}<<0.05$, ${ }^{* *} \mathrm{P}<0.01,{ }^{* * *} \mathrm{P}<0.001$ vs. respective control. ASCL2, achaete-scute homolog 2; OE, overexpression; miR, microRNA.

miR223 is located on the $\mathrm{X}$ chromosome (22). Previous studies have demonstrated that miR223 is associated with tumor and inflammation-associated signal transduction pathways (23). It has been reported that miR223 was highly expressed in patients with acute myeloid leukemia (AML) (22); further studies demonstrated that miR223 served an important role in the regulation of AML (24), although miR223 was downregulated in chronic myelognous leukemia (CLL), and may be associated with tumor burden and tumor invasion. In a previous study, it was observed that ASCL2 may upregulate EMT-associated protein expression to promote the metastasis of GC cells. Therefore, the role of miR223 in GC cells, and the association between ASCL2 and miR223, was the focus of the present study. It was observed that miR223 expression was significantly reduced out of a series of EMT-associated miRNAs. miR223 was able to decrease the migratory ability and downregulate the expression of Zeb-1, Twist, Integrin, Vimentin, MMP-2 and MMP-9 in GC cells; however, in the ASCL2-overexpressing cell line, the decrease in the expression of EMT-associated proteins was improved, thereby suggesting that ASCL2 may affect the role of miRNAs. It was observed that ASCL2 was able to bind to pre-miR223, which inhibited the maturation of miR223 and weakened the biological function of miR223, thus reducing the inhibition of GC cell metastasis.

The present study demonstrated that ASCL2 was able to downregulate the expression level of miR223, contribute to EMT and promote gastric tumor metastasis, indicating that ASCL2 may serve as a therapeutic target in the treatment of
GC. Future studies are required to investigate the association between ASCL2 and other miRNAs, further enriching the molecular mechanism of GC cell invasion and metastasis, and providing a novel effective potential target for $\mathrm{GC}$.

\section{Acknowledgements}

Not applicable.

\section{Funding}

The present study was funding by Innovation project fund of the science and Technology Commission of Putuo (grant no. 2011PTKW003).

\section{Availability of data and materials}

All data generated or analyzed during this study are included in this published article.

\section{Authors' contributions}

QZ performed experimental design, specimen collection and experimental techniques. JW was performed specimen collection and experimental techniques. RZ performed experiment assistance and writing. TC performed experimental design. CC and DXF performed experimental techniques. YZ performed specimen collection. 


\section{Ethics approval and consent to participate}

The procedure was approved by the ethical committee of Putuo Hospital Affiliated to Shanghai University of Traditional Chinese Medicine (Shanghai, China). All patients provided written informed consent.

\section{Consent for publication}

The patient, or parent, guardian or next of kin provided written informed consent for the publication of any associated data and accompanying images.

\section{Competing interests}

The authors declare that they have no conflict of interest.

\section{References}

1. Kwon OH, Park JL, Baek SJ, Noh SM, Song KS, Kim SY and Kim YS: Aberrant upregulation of ASCL2 by promoter demethylation promotes the growth and resistance to 5-fluorouracil of gastric cancer cells. Cancer Sci 104: 391-397, 2013.

2. Pisani P, Parkin DM, Bray F and Ferlay J: Erratum: Estimates of the worldwide mortality from 25 cancers in 1990. Int J Cancer, 83, 18-29 (1999). Int J Cancer 83: 870-873, 1999.

3. Yuasa Y: Control of gut differentiation and intestinal-type gastric carcinogenesis. Nat Rev Cancer 3: 592-600, 2003.

4. Massari ME and Murre C: Helix-loop-helix proteins: Regulators of transcription in eucaryotic organisms. Mol Cell Biol 20: 429-440, 2000.

5. Miyamoto T, Jinno Y, Sasaki T, Ikeda Y, Masuzaki H, Niikawa N and Ishikawa M: Genomic cloning and localization to chromosome 11p15.5 of the human achaete-scute homolog 2 (ASCL2). Cytogenet Cell Genet 73: 312-314, 1996.

6. van der Flier LG, van Gijn ME, Hatzis P, Kujala P, Haegebarth A, Stange DE, Begthel H, van den Born M, Guryev V, Oving I, et al: Transcription factor achaete scute-like 2 controls intestinal stem cell fate. Cell 136: 903-912, 2009.

7. Jubb AM, Chalasani S, Frantz GD, Smits R, Grabsch HI, Kavi V, Maughan NJ, Hillan KJ, Quirke P and Koeppen H: Achaete-scute like 2 (ascl2) is a target of Wnt signalling and is upregulated in intestinal neoplasia. Oncogene 25: 3445-3457, 2006.

8. Zhu R, Yang Y, Tian Y, Bai J, Zhang X, Li X, Peng Z, He Y, Chen L, Pan Q, et al: Ascl 2 knockdown results in tumor growth arrest by miRNA-302b-related inhibition of colon cancer progenitor cells. PLoS One 7: e32170, 2012.

9. Nicoloso MS, Spizzo R, Shimizu M, Rossi S and Calin GA: MicroRNAs-the micro steering wheel of tumour metastases. Nat Rev Cancer 9: 293-302, 2009.

10. Gal H, Pandi G, Kanner AA, Ram Z, Lithwick-Yanai G, Amariglio N, Rechavi G and Givol D: MIR-451 and Imatinib mesylate inhibit tumor growth of Glioblastoma stem cells. Biochem Biophys Res Commun 376: 86-90, 2008.
11. Lee NS, Kim JS, Cho WJ, Lee MR, Steiner R, Gompers A, Ling D, Zhang J, Strom P, Behlke M, et al: miR-302b maintains 'stemness' of human embryonal carcinoma cells by post-transcriptional regulation of Cyclin D2 expression. Biochem Biophys Res Commun 377: 434-440, 2008.

12. Yu F, Yao H, Zhu P, Zhang X, Pan Q, Gong C, Huang Y, Hu X, $\mathrm{Su} F$, Lieberman J and Song E: let-7 regulates self renewal and tumorigenicity of breast cancer cells. Cell 131: 1109-1123, 2007.

13. Jemal A, Siegel R, Ward E, Hao Y, Xu J, Murray T and Thun MJ: Cancer statistics, 2008. CA Cancer J Clin 58: 71-96, 2008.

14. Tsujimoto H, Ono S, Ichikura T, Matsumoto Y, Yamamoto J and Hase K: Roles of inflammatory cytokines in the progression of gastric cancer: Friends or foes? Gastric Cancer 13: 212-221, 2010.

15. Tian Y, Pan Q, Shang Y, Zhu R, Ye J, Liu Y, Zhong X, Li S, He Y, Chen L, et al: MicroRNA-200 (miR-200) cluster regulation by achaete scute-like 2 (Ascl2): Impact on the epithelial-mesenchymal transition in colon cancer cells. J Biol Chem 289: 36101-36115, 2014.

16. Solt LA, Griffin PR and Burris TP: Ligand regulation of retinoic acid receptor-related orphan receptors: Implications for development of novel therapeutics. Curr Opin Lipidol 21: 204-211, 2010

17. Becker-Andre M, Andre E and DeLamarter JF: Identification of nuclear receptor mRNAs by RT-PCR amplification of conserved zinc-finger motif sequences. Biochem Biophys Res Commun 194: 1371-1379, 1993.

18. Schuijers J, Junker JP, Mokry M, Hatzis P, Koo BK, Sasselli V, van der Flier LG, Cuppen E, van Oudenaarden A and Clevers H: Ascl2 acts as an R-spondin/Wnt-responsive switch to control stemness in intestinal crypts. Cell Stem Cell 16: 158-170, 2015.

19. Carlberg C, Hooft van Huijsduijnen R, Staple JK, DeLamarter JF and Becker-André M: RZRs, a new family of retinoid-related orphan receptors that function as both monomers and homodimers. Mol Endocrinol 8: 757-770, 1994.

20. Jetten AM, Kurebayashi S and Ueda E: The ROR nuclear orphan receptor subfamily: Critical regulators of multiple biological processes. Prog Nucleic Acid Res Mol Biol 69: 205-247, 2001.

21. Gawlas K and Stunnenberg HG: Differential transcription of the orphan receptor RORbeta in nuclear extracts derived from Neuro2A and HeLa cells. Nucleic Acids Res 29: 3424-3432, 2001.

22. Chen CZ, Li L, Lodish HF and Bartel DP: MicroRNAs modulate hematopoietic lineage differentiation. Science 303: 83-86, 2004.

23. Stamatopoulos B, Meuleman N, Haibe-Kains B, Saussoy P, Van Den Neste E, Michaux L, Heimann P, Martiat P, Bron D and Lagneaux L: microRNA-29c and microRNA-223 down-regulation has in vivo significance in chronic lymphocytic leukemia and improves disease risk stratification. Blood 113: 5237-5245, 2009.

24. Mi S, Lu J, Sun M, Li Z, Zhang H, Neilly MB, Wang Y, Qian Z, Jin J, Zhang Y, et al: MicroRNA expression signatures accurately discriminate acute lymphoblastic leukemia from acute myeloid leukemia. Proc Natl Acad Sci USA 104: 19971-19976, 2007.

This work is licensed under a Creative Commons Attribution-NonCommercial-NoDerivatives 4.0 International (CC BY-NC-ND 4.0) License. 